\title{
Bridging the Gap Between Single-Strain and Community-Level Plant-Microbe Chemical Interactions
}

\author{
Bridget S. O'Banion, ${ }^{1}$ Lindsey O'Neal, ${ }^{2}$ Gladys Alexandre, ${ }^{2}$ and Sarah L. Lebeis ${ }^{1, \dagger}$ \\ ${ }^{1}$ Department of Microbiology, University of Tennessee, Knoxville, TN, U.S.A. \\ ${ }^{2}$ Department of Biochemistry and Cellular and Molecular Biology, University of Tennessee
}

Accepted 28 October 2019.

\begin{abstract}
Although the influence of microbiomes on the health of plant hosts is evident, specific mechanisms shaping the structure and dynamics of microbial communities in the phyllosphere and rhizosphere are only beginning to become clear. Traditionally, plant-microbe interactions have been studied using cultured microbial isolates and plant hosts but the rising use of 'omics tools provides novel snapshots of the total complex community in situ. Here, we discuss the recent advances in tools and techniques used to monitor plant-microbe interactions and the chemical signals that influence these relationships in above- and belowground tissues. Particularly, we highlight advances in integrated microscopy that allow observation of the chemical exchange between individual plant and microbial cells, as well as highthroughput, culture-independent approaches to investigate the total genetic and metabolic contribution of the community. The chemicals discussed have been identified as relevant signals across experimental spectrums. However, mechanistic insight into the specific interactions mediated by many of these chemicals requires further testing. Experimental designs that attempt to bridge the gap in biotic complexity between single strains and whole communities will advance our understanding of the chemical signals governing plantmicrobe associations in the rhizosphere and phyllosphere.
\end{abstract}

Keywords: bacteria-plant symbiosis, endophytes, microscopy and imaging, rhizosphere and phyllosphere ecology

Plants and their associated microbes dramatically influence each other's lifestyles and health trajectories. Plant tissues provide a source of labile nutrients and niche space to microorganisms, while beneficial microorganisms increase growth, abiotic stress tolerance, and pathogen resistance of their plant host. As each plant microbiome forms, intra- and interkingdom interactions at the tissue interface can influence the overall assemblage and

Current address of L. O'Neal: Department of Microbiology, University of Washington, Seattle, WA, U.S.A.

${ }^{\dagger}$ Corresponding author: S. L. Lebeis; slebeis@utk.edu

Funding: This work was supported by National Science Foundation grants MCB-1715185 to G. Alexandre and IOS-1750717 to S. L. Lebeis. Conclusions or recommendations expressed in this material are those of the authors and do not necessarily reflect the views of the National Science Foundation.

The author(s) declare no conflict of interest.

(c) 2020 The American Phytopathological Society functioning of the microbial community. These interactions are often studied at the extremes of complexity, utilizing molecular and 'omics techniques to investigate either single strains or whole microbial communities (Fig. 1). Although both approaches provide valuable information to the plant-microbe interactions field, it is often difficult to directly link these two ends of the experimental spectrum. Interestingly, however, key chemicals mediating interactions in the rhizosphere (the layer of soil surrounding the root system), root endosphere (internal root tissue), and phyllosphere (aboveground plant surfaces and internal tissues) have been identified by multiple studies, suggesting a strong biological relevance across experimental scales.

Research investigating how individual microbial strains initiate interactions, successfully colonize, and influence the health of their plant hosts is growing. Although these plant-microbe interactions occur on small spatiotemporal scales, their effects are often observed on the whole plant days to weeks after initial contact. Single bacterial strain-plant molecular investigations often overlook the crucial biotic complexity encountered in natural environments. At the microbiome level, huge datasets (e.g., metagenomics and metabolomics) are often generated using homogenized, whole-tissue samples. Although these approaches provide a glimpse into dynamic in situ community interactions, the resulting data are complex and must be approached with targeted hypotheses in order to yield meaningful conclusions, and still often provide only correlative insight. Although large community-level datasets generate hypotheses regarding genetic and chemical targets mediating plant-microbe interactions, strainspecific studies in axenic systems allow definitive characterization of the causative mechanisms. Importantly, these two types of experimental approaches are complementary and often corroborate each other. Thus, the intersection of these approaches facilitates discovery of novel mechanisms and the placement of these findings into a larger biological context. Of increasing interest is the translation of insights from basic plant-microbe research into agricultural practices to improve plant performance in the face of increased abiotic stress. Effectively doing this requires a thorough understanding of the scalability and resilience of interaction mechanisms across ecosystem types.

There is ample information available on chemical exchanges and molecular dialogues that direct the establishment of bacterial and mycorrhizal pathogens or symbionts with host plants. Because these symbiotic (e.g., rhizobia nodulation and mycorrhizal fungi) and pathogenic (which can be considered a form of specialized symbiosis) interactions have been extensively reviewed (Nadal and Paszkowski 2013; Oldroyd 2013; Schmitz and Harrison 2014; Venturi and Fuqua 2013; Venturi and Keel 2016; Via et al. 2016), we will not discuss them in detail here. In contrast, much less is understood about how 
microorganisms that do not strictly fit within either of these categories interact with host plants. These microbes and the communities they form may be beneficial, neutral, or harmful to the plant host under a variety of conditions, the mechanisms of which are generally unknown. Therefore, in this review, we focus on the signals that drive plant-bacteria interactions, rather than the subsequent result of the interaction on host health. Although many critical chemical compounds have been identified, we primarily discuss sugars, amino acids, organic acids, phenolic compounds, and plant hormones, which have been implicated by both single-strain and whole-community approaches. Additionally, we suggest approaches to bridge gaps in experimental designs to study plant-microbe associations in the rhizosphere and phyllosphere at multiple scales.

\section{TOOLS AND TECHNIQUES}

Multiple techniques used to track plant-microbe associations highlight a critical role for chemicals in structuring these associations across spatial and temporal scales. Several mechanisms exist by which plants and microbes influence each other's growth, and understanding how the components of individual interactions combine to result in a whole, dynamic community is only now coming into focus. Recent advances in

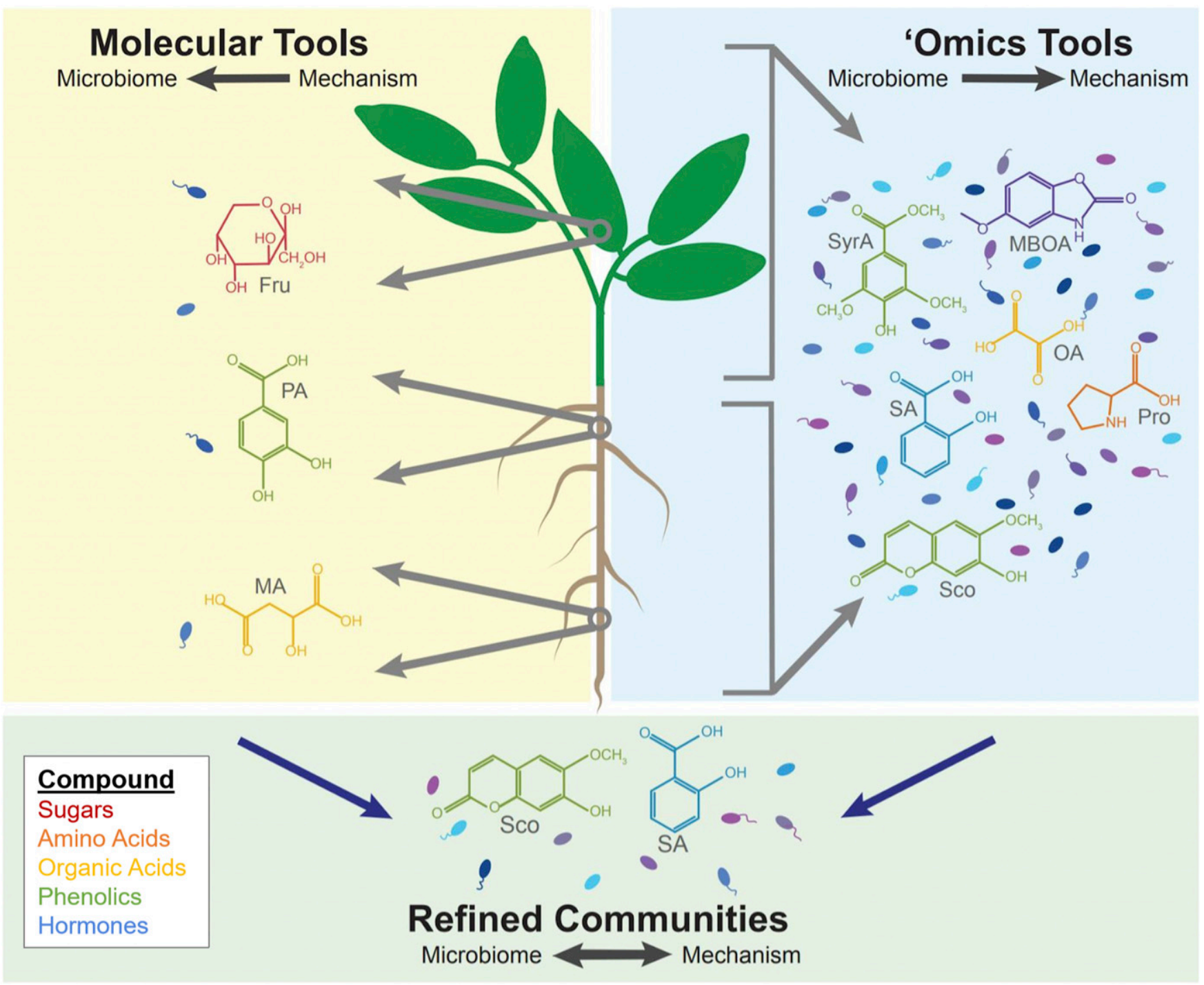

Fig. 1. Chemical signals mediating plant-microbe interactions have been identified using a variety of experimental tools. Many classes of compounds have direct or indirect evidence supporting their role in mediating phenotypes at different spatial scales. Molecular tools such as microscopy and biochemical assays can reveal specific mechanisms between plants and single microbial strains at cellular scales (yellow shading). These interactions are often investigated using a small set of model plant and bacterial systems, which can limit biological relevance to complex environmental systems. Conversely, 'omics tools such as metagenomics and metabolomics provide a glimpse into all interactions occurring within a system at a given sample point (blue shading). Resulting data are often correlative and hypothesis driving but mechanisms facilitating specific interactions are difficult to pin down without additional testing. One potential approach to bridge these two experimental toolsets is to use refined communities (such as synthetic communities or enrichments) of microorganisms (green shading). An advantage to synthetic communities is that microbial isolates, or specific genes within single isolates, can be added to or removed from the community to observe resultant effects. Enrichment communities use a wild inoculum and more broadly encompass the natural functional potential of an ecosystem. Refined communities more accurately capture biotic complexity and emergent effects present in natural systems, while still allowing the tractability and control of a laboratory setting. The compounds drawn in each shaded section represent chemicals that have been specifically identified by different types of experimental approaches and are not exhaustive of the current literature. Chemical structures are colored according to the legend in the white box. Fru $=$ fructose, $\mathrm{PA}=$ protocatechuic acid, MA $=$ malic acid, SyrA $=$ syringic acid, $\mathrm{MBOA}=6$-methoxy-benzoxazolin-2-one (benzoxazinoid breakdown product), $\mathrm{SA}=$ salicylic acid, $\mathrm{OA}=$ oxalic acid, Pro $=$ proline, and $\mathrm{Sco}=$ scopoletin. 
visualizing microscopic interactions and culture-independent techniques to quantify biological and chemical components in a system provide distinct yet complementary insights into rhizosphere and phyllosphere interactions.

\section{Microscopy.}

The ability to directly visualize cellular associations via microscopic techniques allows interactions to be monitored at microbially relevant spatial and temporal scales. Microscopic characterization of plant-microbe associations has benefited from advances in live microscopy, including the development of fluorescent dyes, coupled with microfluidics and spectral analytical methods. Although live microscopy offers clear advantages to visualize how these interactions become established, there are limitations regarding the nature of the processes that can be visualized. On the other hand, microscopic techniques using fixed samples typically reach higher spatial resolution but only allow for end-point analyses. Microscopic techniques are well suited to understand interactions between single microbial strains and single plant hosts, especially when these analyses include comparisons of defined mutants in characterized pathways. In these cases, microscopy is a straightforward method to test specific hypotheses. New combinatorial labeling techniques are beginning to offer insight into simple community dynamics as well. Additionally, techniques tying microscopy to chemical analyses are providing novel insight into metabolites mediating these interactions.

Plant-microbe interactions can be investigated on a microscale through live imaging of fluorescently labeled microbial strains. Studies using this approach have shown chemotactic accumulation of microbes to plant tissue, such as with a $B a$ cillus subtilis strain colonizing Arabidopsis thaliana roots within a few hours (Allard-Massicotte et al. 2016). Recently, the development and application of microfluidic devices allowed short-term uninterrupted and real-time visualization of these interactions. Massalha et al. (2017) used a newly developed system, termed a tracking root interactions system, to reveal discrete steps in the initial interaction between $B$. subtilis and $A$. thaliana. By observing the motility behavior of $B$. subtilis around the roots of A. thaliana inside the microfluidics device, these authors demonstrated that motile cells accumulated in the root elongation zone within $30 \mathrm{~min}$ of bacterial inoculation, followed by a gradual spread across other root regions, presumably through biofilm formation (Massalha et al. 2017).

Although system miniaturization facilitates precise experimental control, it can also impose strict size limits on usable plant systems. The small root systems of $A$. thaliana seedlings are amenable to these small setups but larger, agriculturally relevant crops would likely present technical difficulties. A potential method to overcome the size limitation of microfluidic devices is the use of transparent soil for in situ studies. Artificial soil particles provide an analog for the naturally heterogenous aggregate environment of the soil but with a lowrefractive index suitable for microscopic imaging (Downie et al. 2012). The particles, made with Nafion, allow plant roots to encounter complex physical stimuli and develop similarly to natural soil conditions. The Nafion refractive index is very close to that of an aqueous solution, thus allowing researchers to add plant nutrient solution and image through both the particles and solution to view interactions without light distortion due to refraction. This enables spatial investigations of bacterial strains in vivo and has been used to reveal colonization patterns of Escherichia coli and Pseudomonas fluorescens on Lactuca sativa (lettuce) roots (Downie et al. 2012, 2014).

Live-imaging approaches can also track multiple microbial strains in tandem. For example, Massalha et al. (2017) showed that $B$. subtilis actively excludes $E$. coli from the root surface in dual-inoculation experiments. As long as fluorescent reporters are sufficiently distinct and appropriate filters are available, imaging techniques can separate signals from multiple microbes. Recent work in the oral microbiome successfully used seven different fluorescent dyes to track polymicrobial dynamics over space and time in vitro, which could lend itself to the study of plant-bacteria interactions (Shrivastava et al. 2018). These nontoxic dyes bind to components of the cell such as free amine groups, providing a nondiscriminatory way of fluorescently labeling a population of cells. Because these dyes do not require genetic manipulation, a larger variety of microorganisms can be investigated, beyond genetically tractable lab strains. A major drawback to using nongenetically encoded fluorescence, however, is that cell division dilutes the signal rapidly and thereby restricts the time scales amenable to this approach.

Although microscopic techniques allow researchers to visualize interactions at the cellular level, the chemicals mediating these interactions often remain enigmatic. Fixed samples provide an alternative route to live imaging for probing chemicals that drive plant-microbe interactions. One method to tie metabolites to microscopy is pairing Raman spectroscopy with scanning electron microscopy. Researchers using this technique were able to link the production of lignin, pectin, chlorophyll, and indole acetic acid to interactions between A. thaliana and a Pantoea isolate (Polisetti et al. 2016). Although promising, this technique still faces technical challenges related to minimizing strong root autofluorescent signals, which can only be overcome by aging the plants (Polisetti et al. 2016). In the leaves, researchers face similar problems with autofluorescence of photosynthetic pigments. Leaf fluorescent in situ hybridization corrects for photosynthetic pigment autofluorescence and accounts for the three-dimensional structure of leaves. This technique has been used to demonstrate differences in niche preference and proliferation within a single bacterial genus (Peredo and Simmons 2018). The observed distribution of bacterial cells correlated with chemical distribution results from quantitative nuclear magnetic resonance paired with imaging high-resolution mass spectrometry, revealing that amino acids and sugars were likely used to support microbial growth in leaves (Ryffel et al. 2016).

Another approach for teasing apart the influence of certain chemicals on colonization is to use genetic knockouts and compare wild-type to mutant interaction phenotypes. The tractability of bacterial chemotaxis pathways has made it an insightful model to decipher specific chemicals that motile bacteria respond to in various environments, including plant rhizospheres (Pii et al. 2015; Scharf et al. 2016). Genetic knockouts with altered colonization or plant interaction phenotypes can then be linked to the chemicals related to that specific receptor (Allard-Massicotte et al. 2016). Chemotaxis assays can illuminate which ligands present in root exudates are substrates for specific chemoreceptors (Feng et al. 2018). Although technical progress is evident, increasing both spatial and temporal resolutions of these microscopic and analytical methods is required to gain a realistic understanding of the interplay between the chemistry of plants and their associated microbes.

\section{Comparative genomics and culture-independent approaches.}

The emergence of high-throughput sequencing facilitates large-scale investigations of in situ plant-microbe interactions, even within highly complex communities. Microorganisms that are readily isolated in the laboratory can have their entire genomes sequenced and annotated at rapid and high-quality rates. 
Fully sequenced genomes can then be compared using computational tools to identify conserved and unique traits across different environments, lifestyle, or other metadata categories. Although many members of the plant microbiome can be cultured (Bai et al. 2015), there are still biologically important microorganisms that elude isolation techniques. Therefore, applying techniques such as amplicon and metagenomic sequencing that do not require prior cultivation can help advance the field in a multitude of directions.

Whole-genome analyses of cultured microorganisms can reveal genetic determinants for niche adaptation in the plant environment. Work by Levy et al. (2018) compared the genomes of over 3,800 bacterial isolates to identify genetic factors that may represent adaptation to life in association with a plant host or root system. They found that plant-associated bacterial genomes had increased potential for chemotaxis and carbohydrate metabolism or transport, suggesting that microbes in the plant environment are attracted by metabolizable plantderived compounds. As more and more genomes are sequenced and deposited in public databases, large-scale comparisons will provide an avenue to develop hypotheses regarding interactions between microbes and plant hosts that can then be tested in the laboratory or field.

Culture-independent amplicon sequencing of the $16 \mathrm{~S}$ or $18 \mathrm{~S}$ ribosomal RNA (rRNA) gene and internal transcribed spacer region reveals the archaeal, bacterial, protist, and fungal community composition at each sampling point, which is useful for understanding community-wide responses to plant development stage, exudate composition, and environmental perturbations. However, because these amplicon surveys only allow taxonomic exploration of the community, the functional mechanisms behind community transitions remain unknown. Analyses combining chemical, physiological, and genetic lines of evidence with amplicon datasets can make powerful cases for important mechanisms in the plant environment. Zhalnina et al. (2018) linked 16S rRNA gene sequences, plant exometabolomics, whole-genome sequences, and microbial substrate use to identify chemical patterns in the oat rhizosphere. The researchers found that bacterial taxa with increased relative abundance in the root environment encoded more organic acid transporters and imported more plant-derived amino acids, organic acids, sugars, quaternary amines, and phenolic compounds than bacteria with decreased relative abundance in the root environment (Zhalnina et al. 2018).

Metagenomic sequencing takes the taxonomic investigations of amplicon sequencing a step further by revealing an untargeted subset of all genes present within a system. These studies can provide a snapshot of the overall community genetic potential, or bin metagenome-assembled genomes (MAGs) to understand how specific microbial populations respond to the environment. Metagenomic approaches provide an abundance of data that can be difficult to parse, and it can be challenging to differentiate active microorganisms from dormant members and relic DNA. There are ways to target community sequencing efforts to "active" members, such as through bioorthogonal noncanonical amino acid tagging (BONCAT) and stableisotope probing (SIP). BONCAT and SIP approaches are both based on active microbial members incorporating or transforming chemical compounds that can be traced through time (Singer et al. 2017). Briefly, SIP takes advantage of natural isotopic ratios (often of $\mathrm{C}$ or $\mathrm{N}$ ) to track biotic transformation and incorporation of labeled compounds. BONCAT relies on microorganisms incorporating artificial amino acids and synthesizing them into new proteins (Hatzenpichler et al. 2014). Active microbial members are then identified through densitygradient centrifugation or whole-cell fluorescence approaches, respectively. Although BONCAT has not yet been applied to the plant microbiome, it has proved useful in other environmental systems such as soil (Couradeau et al. 2019), and has the potential to provide novel information about in situ activity. On the other hand, several research groups have combined traditional DNA sequencing techniques with SIP (DNA-SIP) to dissect plant-microbe interactions (Haichar et al. 2016). For example, researchers characterized a Saccharibacteria MAG from wild oat rhizosphere samples that encoded several mechanisms relevant to life in the root-soil interface, including degradation and detoxification of plant-derived phenolic compounds such as salicylic acid (Starr et al. 2018). Very few Saccharibacteria (formerly TM7) strains have been successfully isolated, which limits physiological studies of this phylum. Therefore, culture-independent investigations can provide a deeper understanding of the lifestyle of microorganisms that presently elude cultivation and laboratory manipulation.

Although DNA approaches (such as amplicon sequencing, metagenomics, and DNA-SIP) have been successfully optimized and applied to plant and soil systems, many other in situ approaches dealing with RNA, proteins, and metabolites (metatranscriptomics, metaproteomics, and metabolomics) are still largely limited by soil particle sorption and the inherent complexity of the system. Although these tools are not discussed here, they have the potential to unlock a deluge of data in the context of plant-microbe interactions (Knief et al. 2012; Newman et al. 2016). Hydroponic systems offer a potential work-around for the physical soil environment but cannot fully mimic natural conditions. Future optimization and application of these 'omics tools will allow researchers to move beyond the genetic potential of a community and toward actual gene expression.

\section{CRITICAL CHEMICALS}

As evidence mounts to suggest that chemical exchanges between plants and microbes are the drivers of phyllosphere and rhizosphere productivity, it becomes increasingly important to parse the distinct role of specific signals in mediating interactions. Although a variety of chemical compounds are suggested to influence microbial colonization of plants, we focus here on chemical signals identified across different analytical spectrums, highlighting the scalability of these compounds to reveal meaningful biological interactions. These include broad classes such as sugars, amino acids, organic acids, phenolics, phytohormones, and other stress compounds, focusing on several specific compounds of interest. Additionally, we suggest future directions for targeted chemical approaches.

\section{Sugars.}

Sugars are a labile source of carbon and energy that support the growth of diverse microbes. Therefore, it is likely that sugar compounds have a generic effect in attracting and sustaining microbial communities across a variety of ecosystems. Releasing simple sugars may be beneficial early in plant development to collect a sufficient microbial population from which more selective plant mechanisms may sculpt communities under future environmental conditions. Overall, sugars may play a significant role in rhizosphere and phyllosphere community functioning, because carbohydrates are abundant in both plant root exudates and in discrete pockets on the surface of leaves (Chaparro et al. 2013; Ryffel et al. 2016; Zhalnina et al. 2018).

The relative abundance of sugars in leaves and root exudates varies in response to developmental stage and microbial colonization (Ryffel et al. 2016; Yamada et al. 2016; Yuan et al. 2018; Zhalnina et al. 2018). For example, sucrose and other simple sugars are most abundant in Avena barbata and 
Arabidopsis thaliana root exudates during early developmental stages, decreasing in relative abundance until senescence (Chaparro et al. 2013; Zhalnina et al. 2018). Because sugars are easily utilized by a wide array of microbes, their presence can attract both beneficial and detrimental organisms. In A. thaliana leaves, the abundance of glucose, fructose, and sucrose are significantly correlated with the colonization patterns of commensal Sphingomonas melonis, as well as pathogenic $P$. syringae pv. tomato (Ryffel et al. 2016). A. thaliana plants infected with $P$. syringae pv. tomato decrease the amount of simple sugars released in root exudates and upregulate sugar influx in roots (Yamada et al. 2016; Yuan et al. 2018). This may be a mechanism used by the plant to reduce sugar availability, thereby increasing microbial competition and indirectly defending against pathogen infection.

In the roots, sugar exudates likely act as a mechanism to attract bulk soil microorganisms to the root system. Soil microbes that can sense, move toward, and ultimately exploit these compounds for growth are able to proliferate in the nutrient-rich rhizosphere. Indeed, genes for signal transduction and carbohydrate transport are enriched in plant-associated bacteria relative to their nonplant-associated counterparts (Levy et al. 2018). Additionally, chemotaxis receptors specific for sugars have been identified in soil bacteria, including B. subtilis (galactose) and B. amyloliquefaciens (Feng et al. 2018; Ortega et al. 2017). Although a specific receptor has not yet been identified, Rhizobium leguminosarum bv. vicae is attracted to galactose and mannitol, and the chemotaxis operon that facilitates sugar attraction is also essential for pea plant nodulation (Miller et al. 2007). Despite the abundance of sugars in plant root exudates, there are fewer carbohydrate-specific chemotaxis receptors characterized to date than those sensing amino acids or organic acids (discussed below). One possible explanation is that sugars can also be sensed independently of chemotaxis receptors, via a direct interaction of phosphotransferase systems for sugar enzymes with chemotaxis signaling complexes, as shown previously for $E$. coli (Somavanshi et al. 2016). Initial promotion of diverse plant-microbe relationships through sugar exudation allows plants to generate a pool of microbes from which to preferentially foster their communities via selective chemicals that are present later in development.

\section{Amino acids.}

Amino acids are highly abundant in the rhizosphere at later developmental stages and may potentially influence plant growth and bacterial attraction to the system. Plants undergoing pathogen infection alter their root amino acid exudation patterns. $P$. syringae pv. tomato infection causes A. thaliana to secrete more amino acids and long-chain organic acids, coupled with reduced exudation of simple sugars and short chain organic acids (Yuan et al. 2018). This suggests a potential selection for specific microbial community members, because short-chain compounds may be easier to metabolize by a wider range of microbes and, therefore, offer little selection power to the host plant. Additionally, amino acid exudation is most abundant in Avena barbata vegetative developmental stages (weeks 6 to 9), after the initial recruitment and assembly of the microbiome (Zhalnina et al. 2018).

To be responsive to the dynamic plant environment, microbes must be able to sense extracellular amino acids, presumably via dedicated receptors (Ortega et al. 2017; Wadhams and Armitage 2004). A large repertoire of chemotaxis receptors that directly bind amino acids with a range of specificity and selectivity have been identified in root-associated bacteria (Ortega et al. 2017; Scharf et al. 2016). Many of these receptors recognize and trigger chemotaxis toward several amino acids.
For instance, Sinorhizobium meliloti and B. subtilis colonize Medicago sativa and Arabidopsis thaliana, respectively, and chemoreceptors specific for amino acids found in root exudates were directly implicated in this ability (Allard-Massicotte et al. 2016; Webb et al. 2014; Webb et al. 2016). S. meliloti encodes $\mathrm{McpU}$, a broad amino acid sensor, which must be functional to facilitate root colonization (Webb et al. 2014, 2016). Likewise, $B$. subtilis encodes two amino acid-specific chemoreceptors, $\mathrm{McpA}$ and $\mathrm{McpB}$, that contribute to the successful colonization of A. thaliana (Allard-Massicotte et al. 2016). The kiwifruit leaf pathogen $P$. syringae pv. actinidiae possesses 43 chemoreceptors, and a recent study has found that at least 3 receptors (i.e., PscA, PscB, and PscC) sense a broad range of amino acids (McKellar et al. 2015), though their importance in plant colonization or pathogenicity has not been experimentally demonstrated. Amino acids appear to be critical in shaping specific associations with many microorganisms, and exudate concentrations may be manipulated by the plants to foster specific interactions at critical developmental stages. The widespread distribution of amino acid-specific receptors in plant-associated bacteria provides another indirect line of evidence that these molecules are important in shaping plant-microbe associations.

\section{Organic acids.}

Plants produce organic acids such as tricarboxylic acid to cycle intermediates and to combat cation-anion imbalance (Chang and Roberts 1992; Jones 1998). Bacteria can sense organic acids and use them as carbon substrates and signaling molecules. Organic acids such as malate, citrate, and fumarate are abundant in the rhizosphere of diverse plants and hypothesized to have a more selective influence on root-associated microbial communities than other exuded compounds such as simple sugars (Chaparro et al. 2013). Many microbial genes were annotated as organic acid transporters in bacterial strains isolated from the rhizosphere of Avena barbata (Zhalnina et al. 2018), suggesting that microbes with this functional capacity are able to make a living in the rhizosphere.

Recent studies demonstrated that organic acids play a role in attracting bacteria to several plant species (banana, cucumber, and wheat) by promoting chemotactic responses and subsequent biofilm formation (Liu et al. 2014; Zhang et al. 2013; Zhou et al. 2015). For example, B. amyloliquefaciens NJN-6 chemotaxes toward malate in banana exudates. Although malate promotes chemotaxis, fumarate induces biofilm formation once the bacterium arrives at the root surface (Zhou et al. 2015). Another study focusing on B. amyloliquefaciens SQR9 found that the strain preferentially colonized cucumber plants that were infected with Fusarium oxysporum f. sp. cucumerinum compared with healthy plants. F. oxysporum f. sp. cucumerinum-infected plants exude more citrate and fumarate, which promote $B$. amyloliquefaciens SQR9 chemotaxis and biofilm formation, respectively (Liu et al. 2014). Although dedicated organic acid chemoreceptors have yet to be identified in these plant-colonizing bacterial strains, organic acids seem to be a driving force in root recognition and colonization. Other bacterial species such as $P$. putida F1 and $P$. putida KT2440 possess chemotaxis receptors identified and characterized as directly binding organic acids (McpR and McpS) but their role in sensing exudates is unclear (García et al. 2015; Parales et al. 2013). In addition to being sensed directly by binding to specific regions within chemotaxis receptors, organic acids can also be detected indirectly via their effects on bacterial metabolism, a process termed as energy taxis (Greer-Phillips et al. 2004). Energy taxis receptors monitor redox changes that are produced when the organic acids are catabolized (Schweinitzer and Josenhans 2010; Taylor et al. 1999). The abundance and diversity of organic acid chemotaxis receptors identified in 
bacterial genomes to date (García et al. 2015; Lacal et al. 2010; Ortega et al. 2017; Parales et al. 2013), paired with the strong chemotaxis responses elicited by organic acids in plantcolonizing isolates, underscores their likely role in attracting bacteria to root surfaces. Consistent with this idea, organic acid chemotaxis receptor mutants highlight the importance of organic acid sensing in attracting bacteria to plant roots (Feng et al. 2018; Greer-Phillips et al. 2004).

\section{Phenolic compounds.}

Although plants generate phenolic compounds during growth, their influence on both the host plant and the microbial community members can range from positive to negative based on concentration. For example, cucumber plants can increase syringic acid concentrations in the surrounding soil to levels that result in their own soil sickness (Wang et al. 2018). One study exogenously applied syringic acid to potted cucumber plants and observed decreased Shannon's diversity of the bacterial community. Specifically, Proteobacteria increased in relative abundance while Firmicutes decreased in relative abundance (Wang et al. 2018), suggesting that different types of microbes might be differentially affected by these compounds.

Coumarins are a broad set of molecules secreted by the plant to aid in nutrient mobilization under iron- and phosphatelimiting conditions, with some also possessing antimicrobial properties (Verbon et al. 2017). Recent studies highlight the role of a variety of coumarins in shaping root-associated microbial community structure in Arabidopsis thaliana (Stringlis et al. 2018; Voges et al. 2019). In particular, host scopoletin transport was found to influence microbial community structure under iron-limiting conditions, correlating with an increased abundance of several members of the Bacteroidetes phylum (Stringlis et al. 2018). Catecholic coumarins, including sideretin, fraxetin, and esculetin, were shown by Voges et al. (2019) to alter the composition of a synthetic microbial community in the rhizosphere of A. thaliana. Specifically, the authors hypothesized that the catecholic coumarins had an antimicrobial effect on a dominant Pseudomonas strain, thereby altering the dynamics of the remaining community members. Exogenous application of coumarins restored wildtype community structure phenotypes to coumarin export mutant plant lines (Voges et al. 2019), suggesting a direct role of these compounds in altering microbiome structure.

Although phenolic compounds may have antimicrobial activity toward some microbial taxa, others are attracted to these compounds. Bacterial chemotaxis toward phenolic compounds such as hydroxybenzoate and protocatechuate was discovered in rhizosphere Pseudomonas strains (Krell et al. 2012; Sampedro et al. 2015), indicating that these compounds may also contribute to attracting bacteria to the rhizosphere. Dedicated chemotaxis receptors that directly bind dihydroxybenzoate and hydroxybenzoate were recently characterized in Comamonas testosteroni (Huang et al. 2016), a strain isolated from a wastewater treatment facility. The presence of chemotaxis receptor paralogs specific for dihydroxybenzoate and hydroxybenzoate in $C$. testosteroni suggests that others with similar function might also be present in the soil, including the rootsoil interface. However, whether such paralogs are indeed found in plant-associated bacteria and any related role of chemotaxis toward such phenolic compounds in the establishment of plant-microbe associations remain to be demonstrated.

Several phenolic compounds serve as signaling molecules, attracting microbial symbionts to the rhizosphere of their legume hosts (Aguilar et al. 1988; Caetano-Anollés et al. 1988; Dharmatilake and Bauer 1992). Although it is clear that flavonoids and other phenolic compounds regulate nod gene expression and, thus, the symbiotic association of rhizobia with legumes, the role of these compounds in the initial attraction of these bacteria and other microbes is debated, because no specific chemoreceptor has been identified and specific chemotaxis responses have not been reproducible (Scharf et al. 2016). Because the assays used to initially measure chemotaxis response have notoriously poor sensitivity and reliability, it is necessary to identify dedicated bacterial chemotaxis receptors for flavonoid or phenolic acid inducers. These identifications would provide conclusive evidence that these compounds do, indeed, act as chemoattractants. Additionally, a more comprehensive analysis of how exuded phenolic compounds impact the structure and dynamics of the rhizosphere community is needed to better define their regulatory roles in plant-microbe associations at the level of the microbiome. Most work to date on phenolics has focused in the rhizosphere and, therefore, very little is known about the relative influence of phenolic compounds on chemical crosstalk in the phyllosphere.

\section{Hormones: Ethylene, jasmonic acid, and salicylic acid.}

Plant hormones are synthesized during normal plant development and in response to various abiotic and biotic stressors, and have been shown to modulate the structure of surrounding microbial communities (Bodenhausen et al. 2014; Carvalhais et al. 2019; Lebeis et al. 2015). Plant ethylene perception has been implicated in phyllosphere microbiome assembly while, belowground, salicylic acid influences the root endophytic community (Bodenhausen et al. 2014; Lebeis et al. 2015). Strigolactones are important molecules mediating symbiotic relationships between arbuscular mycorrhizal fungi (AMF) and their host plants. It has been shown that plants mutated in their ability to produce strigolactones have altered fungal communities (beyond just AMF) but do not exhibit changes in the rhizosphere bacterial community (Carvalhais et al. 2019). This suggests that, under the experimental conditions tested, strigolactones do not exert a major influence on bacterial members of the plant microbiome and, therefore, will not be discussed here. Additionally, auxin is a commonly studied phytohormone found to play a role in many plant-microbe interactions. Auxin is interconnected with many other hormonal pathways, which can make it difficult to dissect the direct and indirect roles of auxin specifically (Shigenaga et al. 2017). Due to the abundance of literature available on this topic, we direct readers to one of several reviews (Boivin et al. 2016; Kunkel and Harper 2018; Spaepen and Vanderleyden 2011) and, instead, restrict our focus to those hormones with less experimental data.

Given the important role of plant hormones in development, it is expected that rhizosphere microbes might detect these compounds and adjust their motility accordingly. This assumption prompted the search for chemotaxis responses toward phytohormones. Chemotaxis toward jasmonate by the plant pathogen Dickeya dadantii was suggested to modulate chemoattraction toward vulnerable, wounded plant tissues to facilitate colonization (Antunez-Lamas et al. 2009). Similarly, Pseudomonas chemotaxis to ethylene (Kim et al. 2007) was found by using a concentration gradient. A precursor to ethylene, 1-aminocyclopropane-1-carboxylate, also acts as a strong chemoattractant to $P$. putida UW4 (Li et al. 2019). Although $P$. aeruginosa $\mathrm{PAO} 1, P$. fluorescens, $P$. putida, and $P$. syringae demonstrated chemotaxis in response to ethylene (Kim et al. 2007), direct binding of ethylene to the proposed chemotaxis receptor responsible for sensing ethylene in $P$. aeruginosa, TlpQ, has not been observed (Corral-Lugo et al. 2018). This inconsistency between studies could be caused by nonspecific initial responses or indirect ethylene sensing by TlpQ. 
Several rhizosphere bacterial species (in the genera Bacillus, Brevibacillus, Paenibacillus, Providencia, and Pseudomonas) were attracted to salicylic acid, another plant hormone, in chemotaxis assays ( $\mathrm{Li}$ et al. 2012). On the other hand, chemotaxis away from salicylic acid was recently observed for B. amyloliquefaciens SQR9 in a different spatial gradient assay (Feng et al. 2018), suggesting that salicylic acid might be used to fine tune microbiome composition. Unfortunately, evidence for the chemotaxis response in all of these examples is incomplete, because nonchemotactic control strains were not included or direct evidence of molecular recognition by isolated chemotaxis receptors is lacking.

Without additional evidence for specific chemotaxis responses in gradients of phytohormones, these proposed mechanisms remain inconclusive. Furthermore, increased production of phytohormones in plants is typically associated with additional responses that are usually meant to prevent bacterial associations with plant tissues; thus, a clear rationale for the evolution of specific chemotaxis responses to phytohormones remains undeveloped. Because there are more lines of evidence from 'omics approaches than molecular evidence, the role of plant hormones in dictating interactions might act on time scales that preclude rapid chemotaxis responses or be indirect connections whose signaling networks still need to be characterized.

\section{Other chemical compounds with emerging evidence.}

In response to dynamic abiotic stimuli or microbial colonization, plants produce a variety of chemical compounds and secondary metabolites often associated with stress. For example, quaternary ammonium compounds are made by plants in response to a variety of environmental stresses, and are most abundantly exuded from Avena barbata during plant senescence (Zhalnina et al. 2018). Quaternary amines such as glycine betaine, trigonelline, and choline are exuded by germinating alfalfa seed and attract motile $S$. meliloti to the rhizosphere (Webb et al. 2016). A dedicated chemotaxis receptor senses these quaternary ammonium compounds by direct binding in this species, suggesting this attraction is important in the association of $S$. meliloti with $M$. sativa roots (Webb et al. 2016).

Secondary metabolites often play a role in plant defense from microbes, insects, and herbivores. Benzoxazinoids $(\mathrm{Bx})$ are nitrogenous secondary metabolites found in many cereal crops, especially maize, that have been found to alter rhizosphere microbial community structure and plant defense hormone regulation ( $\mathrm{Hu}$ et al. 2018; Kudjordjie et al. 2019). Hu et al. (2018) showed that Bx exudate patterns altered the microbial community, and that exogenous application of a Bx breakdown product (6-methoxy-benzoxazolin-2-one) was sufficient to restore wild-type community patterns. Molecular studies have also implicated $\mathrm{Bx}$ compounds in the chemotactic response of P. putida KT2440 to maize roots (Neal et al. 2012). The young plants used in two of these studies suggest that Bx may be used as a chemical tool in the early development of the maize rhizosphere microbiome (Kudjordjie et al. 2019; Neal et al. 2012). Characterizing these signal exchanges at greater temporal and spatial resolution in these and other plant hosts beyond maize should be productive in validating or modifying this hypothesis.

The examples discussed here suggest that exudation of certain classes of compounds by the plants might primarily serve to modulate the dynamic selection of bacteria from the rhizosphere in response to plant stress. However, the mechanisms underlying these effects are poorly defined and many gaps remain to be filled. Studies that have pinpointed specific chemical classes as a result of their investigations are summarized in Figure 2. Sugars, amino acids, and organic acids have often been implicated using a variety of approaches. Interestingly, phenolics have been shown to have a range of phenotypic effects on different microbes, highlighting their potential to be used by the plant to fine tune community structure based on specific environmental parameters. Therefore, phenolic compounds warrant further investigations across scales based on the evidence provided here (Fig. 2A). Fixed microscopy, metabolomics, and SIP or BONCAT are underutilized approaches that likely offer a unique approach to investigating the chemical crosstalk between plants and their microbes (Fig. 2B to D). Applying a diverse set of analytical tools to microbial communities at various complexity levels will advance our knowledge of the scalability of observed phenotypes and, ultimately, help to decipher the mechanisms dictating plantmicrobe interactions across scales.

\section{FILLING THE GAPS: BUILDING EXPERIMENTAL BRIDGES}

The chemical crosstalk between plant hosts and their microbial communities is complex and dynamic. Many experimental techniques have been used to investigate specific chemical exchanges and provide ample direct and indirect lines of evidence implicating compounds from diverse chemical classes (Fig. 2). Interestingly, investigations from opposite ends of experimental design spectrums (culture-dependent to culture-independent, and single strains to wild communities) often converge on similar mechanisms, suggesting that they are conserved across a range of biotic complexities. However, much research remains to be conducted to connect the observations in different study systems. Microbial communities that fall in a middle ground between single strains and wild, complex consortia provide a technically feasible system to decipher specific mechanisms while still accounting for the inherent biotic complexity of natural systems. These communities are achieved by enriching for taxa or building them (i.e., synthetic communities [SynComs]) and provide complementary ways to approach this experimental design, which may provide an opportunity to bridge experimental gaps that remain in the field.

Wild microbial communities can be selectively passaged over multiple generations under specific environmental conditions. The resulting enrichment communities often contain many microorganisms with different abundance as compared with the starting community, creating a less diverse environment that can more easily be parsed for causative interactions leading to observed phenotypes. Two studies used this passaging approach to enrich for microbes that alter flowering time in genetically identical host plants (Lu et al. 2018; Panke-Buisse et al. 2015). Both studies serially passaged rhizosphere microbiomes by harvesting the rhizosphere soil of early- or late-flowering plants and using it to grow the next generation of plants. Flowering was proposed to be delayed by certain microbes via their ability to convert tryptophan into indole acetic acid and increased nitrogen availability through nitrification, as demonstrated by enzymatic activity (Lu et al. 2018; Panke-Buisse et al. 2015). Additionally, the resulting community was enriched for microbes that were rare in the starting community (Lu et al. 2018). Together, these passaging studies allowed researchers to link members of indigenous microbial communities to specific metabolites related to an observable plant phenotype. Along with this, they were able to uncover the contribution of rare microbes, which may have been overlooked prior to enrichment, without culturing representatives. In this manner, passaging or enrichment studies provide an avenue for identifying specific players within a wild, complex community that may exert influence under specific environmental conditions.

In contrast to enrichment studies that use wild community starting inoculums, SynCom approaches take advantage of the 
fact that most microbes present in plant microbiomes have cultured representatives (Bai et al. 2015). Microorganisms from various isolate collections can be combined into communities designed to address a variety of research questions. These can include but are not limited to (i) mechanistically testing overarching hypotheses generated in high-complexity systems or previously established macroecological theories, (ii) determining the impact of specific molecular mechanisms in the context of variable biotic complexity, or (iii) establishing the effect of emergent properties of microbial communities on microbiome

\begin{tabular}{|c|c|c|c|c|c|c|}
\hline & Sugars & $\begin{array}{l}\text { Amino } \\
\text { Acids }\end{array}$ & $\begin{array}{c}\text { Organic } \\
\text { Acids }\end{array}$ & $\begin{array}{c}\text { A } \\
\text { Phenolics }\end{array}$ & Hormones & Other \\
\hline \multirow{2}{*}{$\begin{array}{c}\text { Live } \\
\text { Microscopy }\end{array}$} & 1,3 & $1,3,4$ & & & 2,3 & \\
\hline & & & 3 & & & \\
\hline B & & & & & 20 & \\
\hline D & & & & & 21 & \\
\hline \multirow{2}{*}{$\begin{array}{c}\text { Fixed } \\
\text { Microscopy }\end{array}$} & & & & & & \\
\hline & & & & & 5 & \\
\hline \multirow{2}{*}{$\begin{array}{c}\text { Genetic } \\
\text { Knockouts }\end{array}$} & $1,3,22$ & $1,3,4,6,12$ & 3 & & 3 & \\
\hline & & & & & & \\
\hline \multirow{3}{*}{$\begin{array}{cc}\text { Comparativel } \frac{88}{10} \\
\text { Metagenomics } \frac{80}{8000}\end{array}$} & 7 & & & & & \\
\hline & & & & & & \\
\hline & & & & 17 & & \\
\hline \multirow{3}{*}{$\begin{array}{l}\text { Amplicon } \\
\text { Sequencing }\end{array}$} & & & & 8 & 5,9 & \\
\hline & & 10 & 10 & & 11 & \\
\hline & 13 & 13 & 13 & 13,14 & 5 & $13,15,16$ \\
\hline C & 13 & 13 & 13 & 13 & & 13 \\
\hline \multirow{2}{*}{\multicolumn{7}{|c|}{ Metabolomics $\frac{80}{\frac{80}{8000}}$}} \\
\hline & & & & & & \\
\hline - & 18 & 18 & & & 20 & \\
\hline \multirow{2}{*}{\multicolumn{7}{|c|}{ 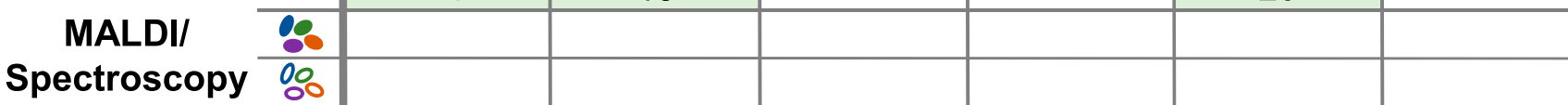 }} \\
\hline \multirow{2}{*}{\multicolumn{7}{|c|}{ Spectroscopy $\frac{100}{\frac{80}{8000}}$}} \\
\hline & & & & & & \\
\hline \multirow{6}{*}{ SIPI BONCAT $\frac{\frac{18}{80}}{800}$} & & & & & & \\
\hline & & & & & & \\
\hline & & & & 19 & 19 & \\
\hline & \multicolumn{4}{|c|}{ Community Complexity } & \multicolumn{2}{|c|}{ Number of Studies } \\
\hline & \multirow{2}{*}{\multicolumn{4}{|c|}{$\begin{array}{l}\text { - Single strain (known) } 002 \text { 2+ strains (unknown) } \\
2+\text { strains (known) } 8000 \text { Wild (unknown) }\end{array}$}} & 1 & \\
\hline & & & & & & \\
\hline
\end{tabular}

Fig. 2. A variety of techniques highlight classes of chemicals driving plant-microbe interactions at different microbial complexities. All chemical classes discussed here have been identified as drivers of interactions across multiple experimental setups (green shaded boxes). There are gaps within the table (empty boxes) that highlight high-priority research areas. For example, A, all chemical classes besides phenolic compounds have been linked to plant-microbe interactions using both culture-dependent and culture-independent techniques listed in the table. Genetic knockouts and amplicon sequencing have been heavily utilized to answer questions of chemical crosstalk between plants and microbes, but $\mathbf{B}$, fixed microscopy has only explicitly linked plant hormones to interactions with microbes. C, Metabolomics has the potential to show catabolism or transformation of a variety of chemical compounds in the context of plant-microbe interactions, as well as changes in exudate composition based on different biotic and abiotic parameters. MALDI = matrix-assisted laser desorption ionization. D, Techniques that target the active portion of the microbial community such as stable-isotope probing (SIP) and bioorthogonal noncanonical amino acid tagging (BONCAT) have the potential to provide an abundance of data in complex communities. Experimental technique categories are subdivided into two to four rows, each representing a microbial complexity ranging from single strains to wild communities. If a technique is not relevant or technically feasible at a specific community complexity, the row for that level has not been included. Solid microbes represent strains that are known to the researchers (isolated or sequenced genomes), while open microbes represent communities that are unknown to the researchers (not cultured or sequenced). Studies are only cited in this table if the results pinpoint specific chemical classes mediating an interaction. References are as follows: $1=$ Allard-Massicotte et al. 2016, $2=$ Antunez-Lamas et al. 2009, $3=$ Feng et al. 2018, $4=$ Webb et al. 2016, $5=$ Lebeis et al. 2015, $6=$ Webb et al. $2014,7=$ Levy et al. $2018,8=$ Voges et al. 2019, 9 = Bodenhausen et al. 2014, 10 = Yuan et al. 2018, 11 = Lu et al. 2018, $12=$ Li et al. 2019, 13= Zhalnina et al. 2018, 14= Wang et al. 2018, $15=$ Kudjordjie et al. 2019, $16=$ Hu et al. 2018, $17=$ Stringlis et al. 2018, $18=$ Ryffel et al. 2016, $19=$ Starr et al. 2018, $20=$ Polisetti et al. $2016,21=$ Peredo and Simmons 2018, and $22=$ Miller et al. 2007. 
functioning. SynComs can be associated with plants under specific environmental conditions and different readouts such as amplicon sequencing or metabolomics can be used to investigate the resulting effects. You can selectively alter the genetic repertoire of a community by swapping out closely related strains, dropping out single strains, or mutating specific genes of interest. Taking this approach to understanding complex community interactions allows specific mechanisms of interest to be investigated. Furthermore, the use of simplified and defined microbial communities will likely improve mechanistic discovery across a range of host-microbe fields.

SynComs are built with a bottom-up approach using a priori knowledge. The set of knowledge used to build each community can vary but typically is based on microbial phylogeny or taxonomy, cooccurrence networks, or functional diversity data from previous plant-microbe sequencing efforts (Vorholt et al. 2017). Although SynComs cannot encapsulate the full diversity of a native soil community, each SynCom selection strategy allows the possibility to test a different set of hypotheses about community composition and functions. Common concerns while building SynComs are which and how many strains to include. Regarding which strains to choose, there is likely no "perfect" combination of microorganisms. The community membership might include a consistent "core" set of microbes and other changing "satellite" microorganisms (Compant et al. 2019). Core microbes are thought to be taxonomically or functionally representative of the typical community found across a variety of plant hosts. Their cosmopolitan presence is often hypothesized to be due to selective pressures commonly imposed by plants, or to consistently beneficial traits imparted by the microbes. However, research is still required to demonstrate that such a community exists and whether all critical members can be cultured in the laboratory. Additionally, satellite members likely play important modulatory roles under different environmental conditions and may be necessary during stressful conditions (Compant et al. 2019). Indeed, because microbiome composition is known to shift in the face of various perturbations, choosing only those microbes found consistently across "wild-type" plants and conditions may bias resulting phenotypes and miss critical members influencing microbiotamediated responses to stressful conditions. Regarding how many strains to include, a balance must be struck between the two ends of the complexity spectra based on the specific research question at hand. The upper limit of the community size is bound by both what is feasible to manage consistently in the laboratory and what is distinguishable by the detection method chosen (e.g., 16S rRNA gene sequencing). Although highly simplified communities $(n \leq 10)$ can much more easily be parsed and manipulated for mechanistic studies, more complex combinations $(n \geq 100)$ likely capture more functional potential (Vorholt et al. 2017). A range of community sizes could be utilized in tandem, and the results of each could be combined and used to create a new community, thereby becoming an iterative process that improves with time and knowledge. Similar approaches have been used to facilitate plant selection of a reduced community from a larger inoculum (Niu et al. 2017).

Understanding how the composition and size of SynComs influence experimental outcomes will be necessary to establish standard guidelines and recommendations for future SynCom design. In the gut microbiome field, a few standardized microbial communities have been put forth as simplified yet still representative communities (Elzinga et al. 2019). However, the implementation of a universal plant SynCom still requires extensive characterization to establish appropriate membership, size, and usefulness to a variety of biological questions. Beyond community composition, there have been recent efforts attempting to standardize other components of plant-microbe studies. Ecosystem Fabrication (EcoFAB) setups attempt to increase reproducibility of biogeochemical parameters across laboratories (Zengler et al. 2019). EcoFABs are a first step to facilitating comparisons of experimental results investigating the plant microbiome across different laboratories.

SynCom have been successfully implemented to reveal both ecological and plant-selective processes occurring during community assembly (Bodenhausen et al. 2014; Voges et al. 2019). Cairns et al. (2018) serially passaged a 33-member community in vitro for 48 days to investigate coexistence and stability over extended time scales. Because the researchers wished to test the overarching ecological question of coexistence, they eliminated the potential influence of coadaptation from similar native environments by choosing SynCom members from a variety of source material, including plants and soils (Cairns et al. 2018). In a plant system, a SynCom built using taxonomic data from amplicon sequencing of wild communities was used to determine that salicylic acid directly and indirectly influences root microbiome composition (Lebeis et al. 2015). Composing SynComs based on microbial functions has been applied to increase $\mathrm{P}_{\mathrm{i}}$ content in the leaves of Arabidopsis thaliana plants grown in phosphate-limited conditions (Herrera Paredes et al. 2018). These examples highlight the variety of methods that can be used to select and combine microbial members into SynComs to answer different questions of interest.

Despite being distinct in scales, molecular and 'omics studies identify numerous chemical classes such as sugars and amino acids that play general roles in attracting and supporting the growth of a wide variety of bacteria. Other compounds such as phenolics and phytohormones may play a more regulatory role in structuring plant-associated microbial communities. However, the specific mechanisms by which bacteria detect and navigate toward or away from plants in response to these signals are generally absent. It may be that bacterial chemotaxis receptors or sensors with these functions are yet to be discovered, or that these chemicals act on established microbial communities indirectly. Clearly, additional analytical methods that track specific chemicals through time and space are needed to fully appreciate the extraordinarily dynamic and complex nature of these environments. Understanding plant-microbe interactions between scales of complexity remains a critical gap that must be filled to advance basic knowledge and to improve the field success of bioinoculants. Although beneficial microbes surely aid in plant growth, those that are developed into agricultural products often have inconsistent success when they are deployed into microbially rich fields (Compant et al. 2019). To understand how the complexity of the biological system contributes to the successes and failures of beneficial microbes in an agricultural setting, it is necessary to establish how the rules of plant-microbe interactions change with dynamic community complexity. Refined communities such as SynComs and enrichment communities represent a possible avenue to bridge these gaps and decipher the chemical signals that underlie the assembly of plant-associated microbial communities (Fig. 1).

\section{LITERATURE CITED}

Aguilar, J. M. M., Ashby, A. M., Richards, A. J. M., Loake, G. J., Watson, M. D., and Shaw, C. H. 1988. Chemotaxis of Rhizobium leguminosarum biovar phaseoli towards flavonoid inducers of the symbiotic nodulation genes. J. Gen. Microbiol. 134:2741-2746.

Allard-Massicotte, R., Tessier, L., Lécuyer, F., Lakshmanan, V., Lucier, J. F., Garneau, D., Caudwell, L., Vlamakis, H., Bais, H. P., and Beauregard, P. B. 2016. Bacillus subtilis early colonization of Arabidopsis thaliana roots involves multiple chemotaxis receptors. MBio 7:e0166416. 
Antunez-Lamas, M., Cabrera, E., Lopez-Solanilla, E., Solano, R. González-Melendi, P., Chico, J. M., Toth, I., Birch, P., Pritchard, L., Liu, H., and Rodriguez-Palenzuela, P. 2009. Bacterial chemoattraction towards jasmonate plays a role in the entry of Dickeya dadantii through wounded tissues. Mol. Microbiol. 74:662-671.

Bai, Y., Müller, D. B., Srinivas, G., Garrido-Oter, R., Potthoff, E., Rott, M., Dombrowski, N., Münch, P. C., Spaepen, S., Remus-Emsermann, M., Hüttel, B., McHardy, A. C., Vorholt, J. A., and Schulze-Lefert, P. 2015. Functional overlap of the Arabidopsis leaf and root microbiota. Nature 528:364-369.

Bodenhausen, N., Bortfeld-Miller, M., Ackermann, M., and Vorholt, J. A. 2014. A synthetic community approach reveals plant genotypes affecting the phyllosphere microbiota. PLoS Genet. 10:e1004283.

Boivin, S., Fonouni-Farde, C., and Frugier, F. 2016. How auxin and cytokinin phytohormones modulate root microbe interactions. Front. Plant Sci. 7:1240.

Caetano-Anollés, G., Crist-Estes, D. K., and Bauer, W. D. 1988 Chemotaxis of Rhizobium meliloti to the plant flavone luteolin requires functional nodulation genes. J. Bacteriol. 170:3164-3169.

Cairns, J., Jokela, R., Hultman, J., Tamminen, M., Virta, M., and Hiltunen, T. 2018. Construction and Characterization of Synthetic Bacterial Community for Experimental Ecology and Evolution. Front. Genet. 9:312.

Carvalhais, L. C., Rincon-Florez, V. A., Brewer, P. B., Beveridge, C. A., Dennis, P. G., and Schenk, P. M. 2019. The ability of plants to produce strigolactones affects rhizosphere community composition of fungi but not bacteria. Rhizosphere 9:18-26.

Chang, K., and Roberts, J. K. 1992. Quantitation of rates of transport, metabolic fluxes, and cytoplasmic levels of inorganic carbon in maize root tips during $\mathrm{K}^{+}$ion uptake. Plant Physiol. 99:291-297.

Chaparro, J. M., Badri, D. V., Bakker, M. G., Sugiyama, A., Manter, D. K. and Vivanco, J. M. 2013. Root exudation of phytochemicals in Arabidopsis follows specific patterns that are developmentally programmed and correlate with soil microbial functions. PLoS One 8:e55731.

Compant, S., Samad, A., Faist, H., and Sessitsch, A. 2019. A review on the plant microbiome: Ecology, functions, and emerging trends in microbial application. J. Adv. Res. 19:29-37.

Corral-Lugo, A., Matilla, M. A., Martín-Mora, D., Silva Jiménez, H., Mesa Torres, N., Kato, J., Hida, A., Oku, S., Conejero-Muriel, M., Gavira, J. A., and Krell, T. 2018. High-affinity chemotaxis to histamine mediated by the TlpQ chemoreceptor of the human pathogen Pseudomonas aeruginosa. MBio 9:e01894-18.

Couradeau, E., Sasse, J., Goudeau, D., Nath, N., Hazen, T. C., Bowen, B. P. Chakraborty, R., Malmstrom, R. R., and Northen, T. R. 2019. Probing the active fraction of soil microbiomes using BONCAT-FACS. Nat. Commun. 10:2770.

Dharmatilake, A. J., and Bauer, W. D. 1992. Chemotaxis of Rhizobium meliloti towards nodulation gene-inducing compounds from alfalfa roots. Appl. Environ. Microbiol. 58:1153-1158.

Downie, H., Holden, N., Otten, W., Spiers, A. J., Valentine, T. A., and Dupuy, L. X. 2012. Transparent soil for imaging the rhizosphere. PLoS One 7:e44276.

Downie, H. F., Valentine, T. A., Otten, W., Spiers, A. J., and Dupuy, L. X. 2014. Transparent soil microcosms allow 3D spatial quantification of soil microbiological processes in vivo. Plant Signal. Behav. 9:e970421.

Haichar, F. E. Z., Heulin, T., Guyonnet, J. P., and Achouak, W. 2016. Stable isotope probing of carbon flow in the plant holobiont. Curr. Opin. Biotechnol. 41:9-13.

Elzinga, J., van der Oost, J., de Vos, W. M., and Smidt, H. 2019. The use of defined microbial communities to model host-microbe interactions in the human gut. Microbiol. Mol. Biol. Rev. 83:e00054-18.

Feng, H., Zhang, N., Du, W., Zhang, H., Liu, Y., Fu, R., Shao, J., Zhang, G., Shen, Q., and Zhang, R. 2018. Identification of chemotaxis compounds in root exudates and their sensing chemoreceptors in plant-growthpromoting rhizobacteria Bacillus amyloliquefaciens SQR9. Mol. PlantMicrobe Interact. 31:995-1005.

García, V., Reyes-Darias, J. A., Martín-Mora, D., Morel, B., Matilla, M. A. and Krell, T. 2015. Identification of a chemoreceptor for $\mathrm{C}_{2}$ and $\mathrm{C}_{3}$ carboxylic acids. Appl. Environ. Microbiol. 81:5449-5457.

Greer-Phillips, S. E., Stephens, B. B., and Alexandre, G. 2004. An energy taxis transducer promotes root colonization by Azospirillum brasilense. J. Bacteriol. 186:6595-6604.

Hatzenpichler, R., Scheller, S., Tavormina, P. L., Babin, B. M., Tirrell, D. A., and Orphan, V. J. 2014. In situ visualization of newly synthesized proteins in environmental microbes using amino acid tagging and click chemistry. Environ. Microbiol. 16:2568-2590.

Herrera Paredes, S., Gao, T., Law, T. F., Finkel, O. M., Mucyn, T., Teixeira, P. J. P. L., Salas González, I., Feltcher, M. E., Powers, M. J., Shank, E. A. Jones, C. D., Jojic, V., Dangl, J. L., and Castrillo, G. 2018. Design of synthetic bacterial communities for predictable plant phenotypes. PLoS Biol. 16:e2003962.

Hu, L., Robert, C. A. M., Cadot, S., Zhang, X., Ye, M., Li, B., Manzo, D., Chervet, N., Steinger, T., van der Heijden, M. G. A., Schlaeppi, K., and Erb, M. 2018. Root exudate metabolites drive plant-soil feedbacks on growth and defense by shaping the rhizosphere microbiota. Nat. Commun. 9:2738.

Huang, Z., Ni, B., Jiang, C. Y., Wu, Y. F., He, Y. Z., Parales, R. E., and Liu, S. J. 2016. Direct sensing and signal transduction during bacterial chemotaxis toward aromatic compounds in Comamonas testosteroni. Mol. Microbiol. 101:224-237.

Jones, D. L. 1998. Organic acids in the rhizosphere-A critical review. Plant Soil 205:25-44.

Kim, H., Kuroda, A., and Takiguchi, N. 2007. Ethylene chemotaxis in Pseudomonas aeruginosa and other Pseudomonas species. Microbes Environ. 22:186-189.

Knief, C., Delmotte, N., Chaffron, S., Stark, M., Innerebner, G., Wassmann, R., von Mering, C., and Vorholt, J. A. 2012. Metaproteogenomic analysis of microbial communities in the phyllosphere and rhizosphere of rice. ISME J. 6:1378-1390.

Krell, T., Lacal, J., Guazzaroni, M. E., Busch, A., Silva-Jiménez, H., Fillet, S., Reyes-Darías, J. A., Muñoz-Martínez, F., Rico-Jiménez, M., GarcíaFontana, C., Duque, E., Segura, A., and Ramos, J. L. 2012. Responses of Pseudomonas putida to toxic aromatic carbon sources. J. Biotechnol. 160:25-32.

Kudjordjie, E. N., Sapkota, R., Steffensen, S. K., Fomsgaard, I. S., and Nicolaisen, M. 2019. Maize synthesized benzoxazinoids affect the host associated microbiome. Microbiome 7:59.

Kunkel, B. N., and Harper, C. P. 2018. The roles of auxin during interactions between bacterial plant pathogens and their hosts. J. Exp. Bot. 69:245-254.

Lacal, J., Alfonso, C., Liu, X., Parales, R. E., Morel, B., Conejero-Lara, F., Rivas, G., Duque, E., Ramos, J. L., and Krell, T. 2010. Identification of a chemoreceptor for tricarboxylic acid cycle intermediates: Differential chemotactic response towards receptor ligands. J. Biol. Chem. 285 23126-23136.

Lebeis, S. L., Paredes, S. H., Lundberg, D. S., Breakfield, N., Gehring, J., McDonald, M., Malfatti, S., Glavina del Rio, T., Jones, C. D., Tringe, S. G., and Dangl, J. L. 2015. Salicylic acid modulates colonization of the root microbiome by specific bacterial taxa. Science 349:860-864.

Levy, A., Salas Gonzalez, I., Mittelviefhaus, M., Clingenpeel, S., Herrera Paredes, S., Miao, J., Wang, K., Devescovi, G., Stillman, K., Monteiro, F., Rangel Alvarez, B., Lundberg, D. S., Lu, T. Y., Lebeis, S., Jin, Z., McDonald, M., Klein, A. P., Feltcher, M. E., Rio, T. G., Grant, S. R., Doty, S. L., Ley, R. E., Zhao, B., Venturi, V., Pelletier, D. A., Vorholt, J. A., Tringe, S. G., Woyke, T., and Dangl, J. L. 2018. Genomic features of bacterial adaptation to plants. Nat. Genet. 50:138-150.

Li, P., Ma, L., Feng, Y. L., Mo, M. H., Yang, F. X., Dai, H. F., and Zhao, Y. X. 2012. Diversity and chemotaxis of soil bacteria with antifungal activity against Fusarium wilt of banana. J. Ind. Microbiol. Biotechnol. 39:1495-1505.

Li, T., Zhang, J., Shen, C., Li, H., and Qiu, L. 2019. 1-Aminocyclopropane1-carboxylate: A novel and strong chemoattractant for the plant beneficia rhizobacterium Pseudomonas putida UW4. Mol. Plant-Microbe Interact 32:750-759. doi:

Liu, Y., Zhang, N., Qiu, M., Feng, H., Vivanco, J. M., Shen, Q., and Zhang, R. 2014. Enhanced rhizosphere colonization of beneficial Bacillus amyloliquefaciens SQR9 by pathogen infection. FEMS Microbiol. Lett. 353:49-56.

Lu, T., Ke, M., Lavoie, M., Jin, Y., Fan, X., Zhang, Z., Fu, Z., Sun, L., Gillings, M., Peñuelas, J., Qian, H., and Zhu, Y. G. 2018. Rhizosphere microorganisms can influence the timing of plant flowering. Microbiome 6:231

Massalha, H., Korenblum, E., Malitsky, S., Shapiro, O. H., and Aharoni, A. 2017. Live imaging of root-bacteria interactions in a microfluidics setup. Proc. Natl. Acad. Sci. U.S.A. 114:4549-4554

McKellar, J. L. O., Minnell, J. J., and Gerth, M. L. 2015. A high-throughput screen for ligand binding reveals the specificities of three amino acid chemoreceptors from Pseudomonas syringae pv. actinidiae. Mol. Microbiol. 96:694-707.

Miller, L. D., Yost, C. K., Hynes, M. F., and Alexandre, G. 2007. The major chemotaxis gene cluster of Rhizobium leguminosarum bv. viciae is essential for competitive nodulation. Mol. Microbiol. 63:348-362.

Nadal, M., and Paszkowski, U. 2013. Polyphony in the rhizosphere: Presymbiotic communication in arbuscular mycorrhizal symbiosis. Curr Opin. Plant Biol. 16:473-479.

Neal, A. L., Ahmad, S., Gordon-Weeks, R., and Ton, J. 2012. Benzoxazinoids in root exudates of maize attract Pseudomonas putida to the rhizosphere. PLoS One 7:e35498. 
Newman, M. M., Lorenz, N., Hoilett, N., Lee, N. R., Dick, R. P., Liles, M. R., Ramsier, C., and Kloepper, J. W. 2016. Changes in rhizosphere bacterial gene expression following glyphosate treatment. Sci. Total Environ. 553:32-41.

Niu, B., Paulson, J. N., Zheng, X., and Kolter, R. 2017. Simplified and representative bacterial community of maize roots. Proc. Natl. Acad. Sci. U.S.A. 114:E2450-E2459.

Oldroyd, G. E. 2013. Speak, friend, and enter: Signalling systems that promote beneficial symbiotic associations in plants. Nat. Rev. Microbiol. 11:252-263.

Ortega, Á., Zhulin, I. B., and Krell, T. 2017. Sensory repertoire of bacterial chemoreceptors. Microbiol. Mol. Biol. Rev. 81:e00033-17.

Panke-Buisse, K., Poole, A. C., Goodrich, J. K., Ley, R. E., and KaoKniffin, J. 2015. Selection on soil microbiomes reveals reproducible impacts on plant function. ISME J. 9:980-989.

Parales, R. E., Luu, R. A., Chen, G. Y., Liu, X., Wu, V., Lin, P., Hughes, J. G., Nesteryuk, V., Parales, J. V., and Ditty, J. L. 2013. Pseudomonas putida $\mathrm{F} 1$ has multiple chemoreceptors with overlapping specificity for organic acids. Microbiology 159:1086-1096.

Peredo, E. L., and Simmons, S. L. 2018. Leaf-FISH: Microscale imaging of bacterial taxa on phyllosphere. Front. Microbiol. 8:2669.

Pii, Y., Mimmo, T., Tomasi, N., Terzano, R., Cesco, S., and Crecchio, C. 2015. Microbial interactions in the rhizosphere : Beneficial influences of plant growth-promoting rhizobacteria on nutrient acquisition process. Biol. Fertil. Soils 51:403-415.

Polisetti, S., Bible, A. N., Morrell-Falvey, J. L., and Bohn, P. W. 2016. Raman chemical imaging of the rhizosphere bacterium Pantoea sp. YR343 and its co-culture with Arabidopsis thaliana. Analyst (Cambridge U.K.) 141:2175-2182.

Ryffel, F., Helfrich, E. J. N., Kiefer, P., Peyriga, L., Portais, J. C., Piel, J., and Vorholt, J. A. 2016. Metabolic footprint of epiphytic bacteria on Arabidopsis thaliana leaves. ISME J. 10:632-643.

Sampedro, I., Parales, R. E., Krell, T., and Hill, J. E. 2015. Pseudomonas chemotaxis. FEMS Microbiol. Rev. 39:17-46. doi:

Scharf, B. E., Hynes, M. F., and Alexandre, G. M. 2016. Chemotaxis signaling systems in model beneficial plant-bacteria associations. Plant Mol. Biol. 90:549-559.

Schmitz, A. M., and Harrison, M. J. 2014. Signaling events during initiation of arbuscular mycorrhizal symbiosis. J. Integr. Plant Biol. 56:250-261.

Schweinitzer, T., and Josenhans, C. 2010. Bacterial energy taxis: A global strategy? Arch. Microbiol. 192:507-520.

Shigenaga, A. M., Berens, M. L., Tsuda, K., and Argueso, C. T. 2017. Towards engineering of hormonal crosstalk in plant immunity. Curr. Opin. Plant Biol. 38:164-172.

Shrivastava, A., Patel, V. K., Tang, Y., Yost, S. C., Dewhirst, F. E., and Berg, H. C. 2018. Cargo transport shapes the spatial organization of a microbial community. Proc. Natl. Acad. Sci. U.S.A. 115:8633-8638.

Singer, E., Wagner, M., and Woyke, T. 2017. Capturing the genetic makeup of the active microbiome in situ. ISME J. 11:1949-1963.

Somavanshi, R., Ghosh, B., and Sourjik, V. 2016. Sugar influx sensing by the phosphotransferase system of Escherichia coli. PLoS Biol. 14:e2000074.

Spaepen, S., and Vanderleyden, J. 2011. Auxin and plant-microbe interactions. Cold Spring Harb. Perspect. Biol. 3:a001438.

Starr, E. P., Shi, S., Blazewicz, S. J., Probst, A. J., Herman, D. J., Firestone, M. K., and Banfield, J. F. 2018. Stable isotope informed genomeresolved metagenomics reveals that Saccharibacteria utilize microbiallyprocessed plant-derived carbon. Microbiome 6:122.

Stringlis, I. A., Yu, K., Feussner, K., de Jonge, R., Van Bentum, S., Van Verk, M. C., Berendsen, R. L., Bakker, P. A. H. M., Feussner, I., and
Pieterse, C. M. J. 2018. MYB72-dependent coumarin exudation shapes root microbiome assembly to promote plant health. Proc. Natl. Acad. Sci. U.S.A. 115:E5213-E5222.

Taylor, B. L., Zhulin, I. B., and Johnson, M. S. 1999. Aerotaxis and other energy-sensing behavior in bacteria. Annu. Rev. Microbiol. 53:103-128.

Venturi, V., and Fuqua, C. 2013. Chemical signaling between plants and plant-pathogenic bacteria. Annu. Rev. Phytopathol. 51:17-37.

Venturi, V., and Keel, C. 2016. Signaling in the rhizosphere. Trends Plant Sci. 21:187-198.

Verbon, E. H., Trapet, P. L., Stringlis, I. A., Kruijs, S., Bakker, P. A. H. M., and Pieterse, C. M. J. 2017. Iron and Immunity. Annu. Rev. Phytopathol. 55:355-375.

Via, V. D., Zanetti, M. E., and Blanco, F. 2016. How legumes recognize rhizobia. Plant Signal. Behav. 11:e1120396.

Voges, M. J. E. E. E., Bai, Y., Schulze-Lefert, P., and Sattely, E. S. 2019 Plant-derived coumarins shape the composition of an Arabidopsis synthetic root microbiome. Proc. Natl. Acad. Sci. U.S.A. 116: 12558-12565.

Vorholt, J. A., Vogel, C., Carlström, C. I., and Müller, D. B. 2017. Establishing causality: Opportunities of synthetic communities for plant microbiome research. Cell Host Microbe 22:142-155.

Wadhams, G. H., and Armitage, J. P. 2004. Making sense of it all: Bacterial chemotaxis. Nat. Rev. Mol. Cell Biol. 5:1024-1037.

Wang, Z., Zhang, J., Wu, F., and Zhou, X. 2018. Changes in rhizosphere microbial communities in potted cucumber seedlings treated with syringic acid. PLoS One 13:e0200007.

Webb, B. A., Helm, R. F., and Scharf, B. E. 2016. Contribution of individual chemoreceptors to Sinorhizobium meliloti chemotaxis towards amino acids of host and nonhost seed exudates. Mol. Plant-Microbe Interact. 29:231-239.

Webb, B. A., Hildreth, S., Helm, R. F., and Scharf, B. E. 2014 Sinorhizobium meliloti chemoreceptor McpU mediates chemotaxis toward host plant exudates through direct proline sensing. Appl. Environ. Microbiol. 80:3404-3415.

Yamada, K., Saijo, Y., Nakagami, H., and Takano, Y. 2016. Regulation of sugar transporter activity for antibacterial defense in Arabidopsis. Science 354:1427-1430.

Yuan, J., Zhao, J., Wen, T., Zhao, M., Li, R., Goossens, P., Huang, Q., Bai, Y., Vivanco, J. M., Kowalchuk, G. A., Berendsen, R. L., and Shen, Q 2018. Root exudates drive the soil-borne legacy of aboveground pathogen infection. Microbiome 6:156.

Zengler, K., Hofmockel, K., Baliga, N. S., Behie, S. W., Bernstein, H. C., Brown, J. B., Dinneny, J. R., Floge, S. A., Forry, S. P., Hess, M., Jackson, S. A., Jansson, C., Lindemann, S. R., Pett-Ridge, J., Maranas, C., Venturelli, O. S., Wallenstein, M. D., Shank, E. A., and Northen, T. R. 2019. EcoFABs: Advancing microbiome science through standardized fabricated ecosystems. Nat. Methods 16:567-571.

Zhalnina, K., Louie, K. B., Hao, Z., Mansoori, N., da Rocha, U. N., Shi, S., Cho, H., Karaoz, U., Loqué, D., Bowen, B. P., Firestone, M. K., Northen, T. R., and Brodie, E. L. 2018. Dynamic root exudate chemistry and microbial substrate preferences drive patterns in rhizosphere microbial community assembly. Nat. Microbiol. 3:470-480.

Zhang, L., Fritsch, M., Hammond, L., Landreville, R., Slatculescu, C., Colavita, A., and Mah, T. F. 2013. Identification of genes involved in Pseudomonas aeruginosa biofilm-specific resistance to antibiotics. PLoS One 8:e61625.

Zhou, G., Yuan, J., and Gao, H. 2015. Regulation of biofilm formation by BpfA, BpfD, and BpfG in Shewanella oneidensis. Front. Microbiol. 6: 790. 